\title{
16. X-RAY MINERALOGY OF SEDIMENTS FROM THE FAR WESTERN PACIFIC, LEG 20, DSDP ${ }^{1}$
}

\author{
J. C. Matti, I. Zemmels, and H. E. Cook, University of California, Riverside
}

\section{INTRODUCTION}

This report synthesizes X-ray mineralogy data from Sites $194,195,196,198$, and 199 of Leg 20 in the far western Pacific. The discussion attempts to characterize the mineralogy of various lithostratigraphic horizons insofar as sample material permits. In view of the intermittent coring, a high degree of sample disturbance, and low core recovery, it is not certain whether the samples are representative of the sedimentary section at the sites drilled. Hence, our conclusions must be viewed as tentative.

Ewing et al. (1968) have identified four acoustic layers in north Pacific deep-sea sediments. These layers are (1) the upper transparent layer, (2) the opaque layer, (3) the lower transparent layer, and (4) the lower opaque layer (also called Horizon B). These sedimentary intervals overlie a rugged, acoustic basement. This generalized acoustic stratigraphy was recognized in the far western Pacific by Leg 20 scientists. One of the objectives of Leg 20 was to obtain a general understanding of the age and lithology of these major acoustic intervals.

Portions of the upper transparent and opaque intervals were drilled at Sites 194, 195, 196, and 198. Shipboard scientists separated the upper transparent interval into two sedimentary suites: (1) a Holocene to late Miocene volcaniclastic silty clay sequence overlying (2) an early Tertiary to Late Cretaceous pelagic clay sequence. The volcaniclastic sediments form a wedge which thins to the east and south, away from the western Pacific island arcs, and which overlaps the pelagic sediments. Shipboard scientists attributed the origin of the volcaniclastic wedge to volcanic arcs in the western Pacific. Sediments from the volcaniclastic suite were X-ray analyzed from Sites 194, 195, and 196. The underlying pelagic clays were sampled for X-ray analyses at Sites 196 and 198.

Pelagic clays of the upper transparent interval gradationally overlie the acoustically opaque interval. The opaque interval was found to consist of Cretaceous clays and calcareous clays frequently interbedded with chert. No samples were submitted for $\mathrm{X}$-ray analyses from the opaque interval, although samples were submitted from the transitional interval between the upper transparent layer and the opaque layer at Site 198. No samples were submitted from the lower transparent or lower opaque intervals.

Site 199, in the Caroline Basin, does not exhibit the characteristic succession of transparent and opaque acoustic layers. Instead, Site 199 consists of a thick sequence of volcanic-ash-rich acoustically transparent sediments. Hence, Site 199 is discussed apart from other Leg 20 sites.

\footnotetext{
${ }^{1}$ Institute of Geophysics and Planetary Physics, University of California, Riverside, Contribution No. 73-19.
}

\section{METHODS}

Semiquantitative determinations of the mineral composition of bulk samples, $2-20 \mu \mathrm{m}$, and $<2 \mu \mathrm{m}$ fractions were performed according to the methods described in the reports of Legs 1 and 2 and in Appendix III of Volume IV. The mineral analyses of the $2-20 \mu \mathrm{m}$ and $<2 \mu \mathrm{m}$ fractions were performed on $\mathrm{CaCO}_{3}$-free residues.

The results are presented in Tables 3 through 7. Sediment ages, lithologic units, and textural and compositional nomenclature of the sediment types in Tables 1 and 2 are from the DSDP Leg 20 Hole Summaries. Samples submitted for X-ray diffraction analyses from Leg 20 are listed in Table 2. The sample depths below the sea floor in the last column of Table 2 identify the samples as they are reported in Tables 3 through 7. No samples were submitted for X-ray diffraction analyses from Sites 197, 200,210, and 202, primarily due to poor recovery of core at these sites.

Several unidentified minerals were detected in Leg 20 samples. Their abundances were determined on a semiqualitative basis using a hypothetical mineral concentration factor of 3.0. Unidentified minerals are reported on a ranked, semiqualitative scale as outlined below:

Trace: $(<5 \%)$; diffraction pattern was weak and identification was made on the basis of two major diagnostic peaks.

Present: (5-25\%); a number of peaks of the mineral are visible in the diffraction pattern.

Abundant: (25-65\%); diffraction peaks of the mineral are prominent in the total diffraction pattern, but the peaks of other minerals are of an equivalent intensity.

Major: $(>65 \%)$; the diffraction peaks of the mineral dominate the diffraction pattern.

Although a certain quantity of the unidentified minerals is implied, their concentration is not included in the concentrations of identified minerals, which are summed to $100 \%$.

The terms "Trace," "Present," etc., are also used in discussing identified minerals in the text of this report and imply the concentration ranges as indicated above.

The use of drilling mud containing montmorillonite and barite on Leg 20 was as follows: Hole 194, during Core 5; Hole 195 between Cores 2 and 3 and between Cores 3 and 4; Hole 195B, after Core 1; Hole 198A before Cores 5 and 6 . The samples which were submitted for X-ray diffraction analyses were not exposed to drilling mud.

\section{RESULTS AND DISCUSSION}

\section{Sites 194 and 195}

Sites 194 and 195 are located on the abyssal ocean floor approximately $450 \mathrm{~km}$ east of the Izu-Bonin Trench. Only 
the volcaniclastic portion of the upper transparent interval was sampled at Sites 194 and 195.

The mineralogy of the bulk, $2-20 \mu \mathrm{m}$, and $<2 \mu \mathrm{m}$ samples at both sites is strikingly similar (Tables 3 and 4 ). The bulk samples of all cores contain primarily mica, quartz, and plagioclase, with subordinate amounts of montmorillonite and chlorite. Quartz predominates over plagioclase in all size fractions. Mica generally predominates in the $<2 \mu \mathrm{m}$ fractions, but montmorillonite predominates over mica in a few samples. Kaolinite appears as traces in the bulk samples of late Miocene sediments, but was not detected in bulk samples of the Quaternary sediments. Kaolinite is more uniformly distributed in $<2 \mu \mathrm{m}$ fractions of most samples, however. Neither calcite nor zeolites were detected in the volcaniclastic sediments.

Colorless, volcanic glass shards commonly constitute $50 \%$ of the lithogenous components. The refractive index of fresh, unaltered glass is between 1.510 and 1.512 , which closely corresponds to the mean refractive indexes of dacite and andesite glass (1.511 and 1.512, respectively, as given by Wahlstrom, 1955).

Cores from Sites 194 and 195 are essentially indistinguishable on the basis of X-ray mineralogy. The sediments are characterized by a mica-quartz-plagioclasemontmorillonite suite containing abundant volcanic glass and traces of amphibole and kaolinite. The relative abundances of the dominant minerals vary between size classes.

\section{Site 196}

Site 196 is located on the abyssal ocean floor approximately $530 \mathrm{~km}$ east of the Izu-Bonin Trench. The volcaniclastic suite and the underlying pelagic suite of the upper transparent interval were sampled. Hence, Site 196 permits us to compare the mineralogy of these two distinct sedimentary suites within a single stratigraphic section.

One sample of Quaternary ash-bearing silty clays from Core 1 (Table 5) consists primarily of mica, quartz, plagioclase, and colorless volcanic glass with minor amounts of montmorillonite and chlorite. Calcite and zeolites were not detected. Core 1 is mineralogically correlative with samples from the volcaniclastic wedge at Sites 194 and 195.

Core 2 sampled Late Cretaceous zeolitic clays from the pelagic portion of the upper transparent interval. The five samples have a generally uniform composition (Table 5). The dominant mineral in the bulk and $<2 \mu$ m samples is palygorskite. Potassium feldspar, phillipsite, and clinoptilolite are major constituents and predominate over quartz, mica, and plagioclase. Montmorillonite predominates over mica in the $<2 \mu \mathrm{m}$ fractions.

\section{Site 198}

Site 198 is located on the abyssal ocean floor approximately $1170 \mathrm{~km}$ east of the Izu-Bonin Trench and $130 \mathrm{~km}$ north of Marcus Island. Samples were submitted from the pelagic portion of the upper transparent interval and from the transitional interval between the upper transparent layer and the underlying opaque layer.

Cores 1, 3 and 4 (Table 6) are Late Cretaceous and consist of brown, zeolitic clays. The samples are mineralogically uniform. Palygorskite, quartz, mica, and potassium feldspar predominate in the bulk samples. Clinoptilolite and phillipsite are present.

Lowermost samples from Core 4 were taken from the transition interval between the upper transparent layer and the opaque layer. These transitional samples are enriched in quartz, cristobalite, and tridymite (Table 6). Microscopic examination revealed a few single, isolated grains of angular silt-sized quartz. Instead, the samples consist predominantly of volcanic glass shards and radiolarian skeletal remains which have been replaced by cristobalite (refractive index 1.484). Many of these shards and skeletal tests display a first-order yellow core surrounded by cristobalite. We attribute the first-order yellow birefringence to quartz and interpret the quartz and enclosing cristobalite to be devitrified products of volcanic glass and radiolarian tests. Tridymite is probably also an authigenic devitrification product.

\section{Site 199}

Site 199 is located on the east margin of the Caroline Abyssal Plain approximately $450 \mathrm{~km}$ north of the Caroline Islands and $1000 \mathrm{~km}$ east of the Marianas Trench. Coring was fairly frequent, and the X-ray diffraction results from Cores 1 to 6 are probably representative of the mineralogy of the sedimentary section at Site 199.

Shipboard scientists recognized eight informal lithologic units at Site 199. Samples from Units A, B, C, D, and E were submitted for X-ray diffraction analyses. The lithology and ages of these units and the major mineral components of the bulk, $2-20 \mu \mathrm{m}$, and $<2 \mu \mathrm{m}$ fractions are summarized in Table 1 and are also presented in Table 7.

Data from Tables 1 and 7 indicate that $\mathrm{CaCO}_{3}$-free silt and clay fractions from lithologic Units A, B, C, and E contain a phillipsite-augite-magnetite-plagioclase-montmorillonite mineral suite which shows only slight proportional variation stratigraphically. This dominant mineral suite is also present in most bulk samples from each lithologic unit, but the lithogenous components tend to be diluted in samples with large amounts of biogenous calcite (particularly in Unit A). Lithologic Units A, B, C, and E are essentially indistinguishable on the basis of X-ray mineralogy of silt and clay fractions. We conclude that Units A, $\mathrm{B}, \mathrm{C}$, and $\mathrm{E}$ are part of a volcanic-rich, mafic-zeolitic-clay sequence in which biogenous calcite occasionally masks the lithogenous components in bulk samples (as in Units A and E).

Lithologic Unit D is easily distinguished from the other lithologic units at Site 199 by the absence of mafics, low percentage of zeolites, and predominance of montmorillonite.

The acoustically opaque interval was penetrated at Site 199 according to Leg 20 Hole Summaries. However, X-ray mineralogy samples from the opaque interval were not submitted to the U.C. Riverside X-ray facility. Okada and Tomita (this volume) report that the Paleocene-Late Cretaceous opaque interval at Site 199 consists of a calcareous clinoptilolite-montmorillonite-cristobalite pelagic clay suite. Thus, the opaque interval at Site 199 may be mineralogically correlative with our samples of the Late Cretaceous opaque interval in Core 4 at Site 198. 


\section{SUMMARY AND CONCLUSIONS}

Leg 20 scientists have demonstrated that the upper transparent interval (Ewing et al., 1968) in the far western Pacific consists of a Holocene-late Miocene volcaniclastic wedge which overlies a Paleogene-Late Cretaceous pelagic sequence of calcareous zeolitic clays. The volcaniclastic wedge was probably derived from the adjacent Japan, Izu-Bonin, and Marianas volcanic arcs since these sediments pinch out eastward away from the arc system.

The volcaniclastic wedge and underlying pelagic sequence are each characterized by a distinct suite of minerals, and are easily distinguished from each other on the basis of X-ray mineralogy. The volcaniclastic wedge was recognized at Sites 194, 195, and 196, and the underlying pelagic suite was recognized at Sites 196 and 198.

X-ray mineralogy studies of 18 samples from Sites 194, 195 , and 196 indicate that the volcaniclastic wedge is characterized by a non-zeolitic, mica-quartz-plagioclasemontmorillonite mineral suite containing abundant unaltered volcanic glass and little biogenous calcite. X-ray mineralogy results from the volcaniclastic wedge reported by Okada and Tomita (this volume) support the results of this paper.

Fresh glass shards in the volcaniclastic wedge have mean refractive indexes of dacite and andesite glass. Hence, glass in the wedge was probably derived from dacitic-andesitic volcanoes. The volcaniclastic wedge is also characterized by abundant, angular, silt-sized quartz grains. If a significant portion of this quartz was derived from volcanic arcs, then silica-oversaturated volcanic source terrains would normally be required. The presence of abundant acidic glass suggests that a volcanic origin for a significant portion of the quartz is possible. Intermediate-acidic calc-alkaline volcanism characterizes the western Pacific volcanic arcs (Dickinson, 1968).

X-ray mineralogy studies of 13 samples from Sites 196 and 198 indicate that the Paleogene-Late Cretaceous pelagic clay sequence underlying the volcaniclastic wedge is characterized by a zeolitic palygorskite-quartz-mica-potassium feldspar mineral suite containing variable amounts of biogenous calcite. Phillipsite is the dominant zeolite, with clinoptilolite subordinate. X-ray mineralogy results from the pelagic clay sequence reported by Okada and Tomita (this volume) concur with the results in this paper.

The pelagic clay sequence is gradational with the underlying acoustically opaque interval at Site 198 (and Sites 195 and 199, Okada and Tomita, this volume). The opaque interval is mineralogically similar to the overlying pelagic clay sequence, but is distinguished by the presence of cristobalite and tridymite. We suggest that these silica phases are authigenic products resulting from devitrification of siliceous glass and radiolarian tests.

X-ray mineralogy studies of 30 samples from Site 199 in the Caroline Basin indicate that the Early Pliocene-Middle Miocene sequence is characterized by a phillipsite-maficsplagioclase-montmorillonite suite. The mineral ratios do not change appreciably throughout the stratigraphic section. Lithologic Units A to E at Site 199 are part of a volcanic-rich, zeolitic clay sequence in which biogenous calcite occasionally masks the lithogenous components.

The lithology and X-ray mineralogy of the upper transparent interval at Site 199 are generally uniform and reflect a mineralogic history which differs from the history of the upper transparent interval at Sites 194, 195, 196, and 198. At the latter sites the upper transparent interval consists of two distinct sediment suites, while at Site 199 the upper transparent interval consists of a single, uniform sedimentary suite.

\section{REFERENCES}

Dickinson, W. R., 1968. Circum-Pacific andesite types: J. Geophys. Res., v. 73, p. 2261-2269.

Ewing, J., M. Ewing, T. Aitken, and W. J. Ludwig, 1968. North Pacific sediment layers measured by seismic profiling. In Knopoff, L., Drake, C. L., and Hart, P. J. (Eds.), The crust and upper mantle of the Pacific area: Am. Geophys. Union, Monogr. 12, p. 147-173.

Wahlstrom, E. E., 1955. Petrographic mineralogy: New York (John Wiley and Sons). 
TABLE 1

Summary of X-Ray Diffraction Results, Site 199, Caroline Basin

\begin{tabular}{|c|c|c|c|c|c|c|c|c|c|c|c|c|}
\hline \multirow[b]{2}{*}{ Core } & \multirow{2}{*}{$\begin{array}{l}\text { Sample } \\
\text { Depth } \\
(\mathrm{m})\end{array}$} & \multirow{2}{*}{$\begin{array}{l}\text { Lithologic Unit } \\
\text { and/or Sediment } \\
\text { Description }\end{array}$} & \multirow[b]{2}{*}{ Age } & \multicolumn{3}{|c|}{$\begin{array}{c}\text { Bulk Sample } \\
\text { Major Constituent }\end{array}$} & \multicolumn{3}{|c|}{$\begin{array}{l}\text { 2-20 } \mu \mathrm{m} \text { Fraction } \\
\text { Major Constituent }\end{array}$} & \multicolumn{2}{|c|}{$\begin{array}{c}<2 \mu \text { m Fraction } \\
\text { Major } \\
\text { Constituent }\end{array}$} & \multirow[b]{2}{*}{ Comments } \\
\hline & & & & 1 & 2 & 3 & 1 & 2 & 3 & 1 & 2 & \\
\hline $\begin{array}{l}1 \\
1 \\
1 \\
1 \\
1 \\
1\end{array}$ & $\begin{array}{l}588 \\
596 \\
60.5 \\
60.6 \\
628 \\
63.1 \\
\end{array}$ & $\begin{array}{l}\text { Unit A } \\
\text { Alternating zeo- } \\
\text { litic clay and } \\
\text { nannofossil ooze }\end{array}$ & 总 & $\begin{array}{l}\text { Calc. } \\
\text { Calc. } \\
\text { Phil. } \\
\text { Calc. } \\
\text { Phil. } \\
\text { Calc. }\end{array}$ & $\begin{array}{l}\text { Plag. } \\
\text { Plag. }\end{array}$ & $\begin{array}{l}\text { Mafi. }^{a} \\
\text { Mafi. }\end{array}$ & $\begin{array}{l}\text { Phil } \\
\text { Phil. } \\
\text { Mafi. } \\
\text { Phil. } \\
\text { Phil. } \\
\text { Phil }\end{array}$ & $\begin{array}{l}\text { Plag. } \\
\text { Mafi. } \\
\text { Phil. } \\
\text { Mafi. } \\
\text { Mafi. } \\
\text { Mafi. }\end{array}$ & $\begin{array}{l}\text { Mafi } \\
\text { Plag. } \\
\text { Plag. } \\
\text { Plag. } \\
\text { Plag. } \\
\text { Plag. }\end{array}$ & $\begin{array}{l}\text { Phil. } \\
\text { Mont. } \\
\text { Mont. } \\
\text { Mont. } \\
\text { Mont. } \\
\text { Phil. }\end{array}$ & $\begin{array}{l}\text { Mont. } \\
\text { Phil. } \\
\text { Phil. } \\
\text { Phil. } \\
\text { Phil. } \\
\text { Mont. }\end{array}$ & $\begin{array}{l}\text { Calcite dilutes } \\
\text { lithogenous com- } \\
\text { ponents in most } \\
\text { samples; phillip- } \\
\text { site without } \\
\text { clinoptilolite. }\end{array}$ \\
\hline $\begin{array}{l}1 \\
1 \\
1 \\
3 \\
3 \\
3 \\
3 \\
\end{array}$ & $\begin{array}{l}64.1 \\
68.0 \\
70.6 \\
77.8 \\
79.9 \\
81.8 \\
82.8 \\
\end{array}$ & $\begin{array}{c}\text { Unit B } \\
\text { Zeolitic clay }\end{array}$ & 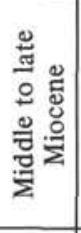 & $\begin{array}{l}\text { Phil. } \\
\text { Phil. } \\
\text { Phil. } \\
\text { Phil. } \\
\text { Phil. } \\
\text { Phil. } \\
\text { Phil. }\end{array}$ & $\begin{array}{l}\text { Plag. } \\
\text { Mafi. } \\
\text { Mafi. } \\
\text { Mafi. } \\
\text { Plag. } \\
\text { Mafi. } \\
\text { Mafi. }\end{array}$ & $\begin{array}{l}\text { Mafi. } \\
\text { Plag. } \\
\text { Plag. } \\
\text { Mont. } \\
\text { Mafi. } \\
\text { Plag. } \\
\text { Mont. }\end{array}$ & $\begin{array}{l}\text { Phil. } \\
\text { Phil. } \\
\text { Phil. } \\
\text { Phil. } \\
\text { Phil. } \\
\text { Phil } \\
\text { Phil. }\end{array}$ & $\begin{array}{l}\text { Mafi. } \\
\text { Mafi. } \\
\text { Mafi. } \\
\text { Mafi. } \\
\text { Mafi. } \\
\text { Mafi. } \\
\text { Mont. }\end{array}$ & $\begin{array}{l}\text { Plag. } \\
\text { Plag. } \\
\text { Plag. } \\
\text { Mont. } \\
\text { Plag. } \\
\text { Mont. } \\
\text { Mafi. }\end{array}$ & $\begin{array}{l}\text { Mont. } \\
\text { Mont. } \\
\text { Mont. } \\
\text { Mont. } \\
\text { Mont. } \\
\text { Mont. } \\
\text { Mont. }\end{array}$ & $\begin{array}{l}\text { Phil. } \\
\text { Phil. } \\
\text { Phil. } \\
\text { Phil. } \\
\text { Phil. } \\
\text { Phil. } \\
\text { Phil. }\end{array}$ & $\begin{array}{l}\text { No calcite; phil- } \\
\text { lipsite and clin- } \\
\text { optilolite occur } \\
\text { together. }\end{array}$ \\
\hline $\begin{array}{l}4 \\
4 \\
4 \\
4 \\
4\end{array}$ & $\begin{array}{l}88.5 \\
88.6 \\
91.2 \\
92.5 \\
93.0\end{array}$ & $\begin{array}{l}\quad \text { Unit C } \\
\text { Zeolitic silty } \\
\text { clay }\end{array}$ & 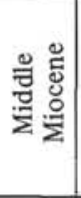 & $\begin{array}{l}\text { Phil. } \\
\text { Phil. } \\
\text { Phil. } \\
\text { Calc. } \\
\text { Calc. }\end{array}$ & $\begin{array}{l}\text { Mafi. } \\
\text { Mafi. } \\
\text { Mafi. } \\
\text { Phil. } \\
\text { Phil. }\end{array}$ & $\begin{array}{l}\text { Mont. } \\
\text { Plag. } \\
\text { Mont. } \\
\text { Mont. } \\
\text { Mont. }\end{array}$ & $\begin{array}{l}\text { Phil. } \\
\text { Phil. } \\
\text { Phil. } \\
\text { Phil. } \\
\text { Phil. }\end{array}$ & $\begin{array}{l}\text { Mafi. } \\
\text { Mafi } \\
\text { Mafi. } \\
\text { Mafi. } \\
\text { Mafi. }\end{array}$ & $\begin{array}{l}\text { Plag. } \\
\text { Plag. } \\
\text { Mont } \\
\text { Plag. } \\
\text { Mont. }\end{array}$ & $\begin{array}{l}\text { Mont. } \\
\text { Phil. } \\
\text { Mont. } \\
\text { Mont. } \\
\text { Mont. }\end{array}$ & $\begin{array}{l}\text { Phil. } \\
\text { Mont. } \\
\text { Phil. } \\
\text { Phil } \\
\text { Phil. }\end{array}$ & $\begin{array}{l}\text { Calcite dilutes } \\
\text { lithogenous com- } \\
\text { ponents in some } \\
\text { samples; phillip- } \\
\text { site without } \\
\text { clinoptilolite. }\end{array}$ \\
\hline $\begin{array}{l}5 \\
5 \\
5 \\
5 \\
5\end{array}$ & $\begin{array}{l}144.1 \\
145.5 \\
148.3 \\
149.8 \\
151.4\end{array}$ & $\begin{array}{c}\text { Unit D } \\
\text { Radiolarian ooze }\end{array}$ & 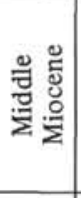 & $\begin{array}{l}\text { Mont. } \\
\text { Mont. } \\
\text { Mont. } \\
\text { Mont. } \\
\text { Mont. }\end{array}$ & $\begin{array}{l}\text { Plag. } \\
\text { Plag. } \\
\text { Plag. } \\
\text { Plag. } \\
\text { Plag. }\end{array}$ & $\begin{array}{l}\text { Phil. } \\
\text { Phil. } \\
\text { Phil. } \\
\text { Phil. } \\
\text { Phil. }\end{array}$ & $\begin{array}{l}\text { Mont. } \\
\text { Mont. } \\
\text { Mont. } \\
\text { Mont. } \\
\text { Mont. }\end{array}$ & $\begin{array}{l}\text { Plag. } \\
\text { Plag. } \\
\text { Plag. } \\
\text { Plag. } \\
\text { Plag. }\end{array}$ & $\begin{array}{l}\text { Phil. } \\
\text { Phil. } \\
\text { Phil. } \\
\text { Phil. } \\
\text { Phil. }\end{array}$ & $\begin{array}{l}\text { Mont. } \\
\text { Mont. } \\
\text { Mont. } \\
\text { Mont. } \\
\text { Mont. }\end{array}$ & & $\begin{array}{l}\text { No mafics; mont- } \\
\text { morillonite domi- } \\
\text { nates in bulk } \\
\text { samples; phillip- } \\
\text { site is minor in } \\
\text { bulk. }\end{array}$ \\
\hline $\begin{array}{l}6 \\
6 \\
6 \\
6 \\
6 \\
6 \\
6\end{array}$ & $\begin{array}{l}200.5 \\
201.0 \\
202.4 \\
202.6 \\
203.6 \\
205.2 \\
206.8\end{array}$ & $\begin{array}{l}\text { Unit E } \\
\text { Interlayered tuff, } \\
\text { nanno-rich clay, } \\
\text { and zeolitic, silty } \\
\text { clay }\end{array}$ & 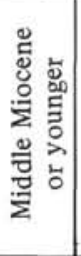 & $\begin{array}{l}\text { Mont. } \\
\text { Phil. } \\
\text { Phil. } \\
\text { Phil. } \\
\text { Phil. } \\
\text { Mont. } \\
\text { Phil. }\end{array}$ & $\begin{array}{l}\text { Phil. } \\
\text { Calc. } \\
\text { Mont. } \\
\text { Plag. } \\
\text { Mont. } \\
\text { Phil. } \\
\text { Mont. }\end{array}$ & $\begin{array}{l}\text { Mont. } \\
\text { Mafi. } \\
\text { Calc. } \\
\text { Mafi. } \\
\text { Mafi. }\end{array}$ & $\begin{array}{l}\text { Phil. } \\
\text { Mont. } \\
\text { Phil. } \\
\text { Phil. } \\
\text { Phil. } \\
\text { Phil. } \\
\text { Phil. }\end{array}$ & $\begin{array}{l}\text { Mont. } \\
\text { Mafi. } \\
\text { Mafi. } \\
\text { Mafi. } \\
\text { Mont. } \\
\text { Mont. } \\
\text { Mafi. }\end{array}$ & $\begin{array}{l}\text { Mafi. } \\
\text { Plag. } \\
\text { Mont. } \\
\text { Mafi. } \\
\text { Mafi. }\end{array}$ & $\begin{array}{l}\text { Mont. } \\
\text { Mont. } \\
\text { Mont. } \\
\text { Mont. } \\
\text { Mont. } \\
\text { Mont. } \\
\text { Mont. }\end{array}$ & $\begin{array}{l}\text { Phil. } \\
\text { Phil. } \\
\text { Phil. } \\
\text { Phil. } \\
\text { Phil. } \\
\text { Phil. }\end{array}$ & $\begin{array}{l}\text { Calcite present; } \\
\text { lithogenous com- } \\
\text { ponents show } \\
\text { greater variability } \\
\text { than in Units } \\
\text { A-D; phillipsite } \\
\text { without } \\
\text { clinoptilolite. }\end{array}$ \\
\hline
\end{tabular}

${ }^{\mathrm{a}}$ Mafi. = mafics: augite and magnetite. Augite always predominates over magnetite, by as much as $5: 1$. 
TABLE 2

Sediment Samples Submitted for X-Ray Diffraction Analysis

Depth in Section Depth Below Sea Floor

Core, Section

(cm) (m)

Hole 194

$\begin{array}{lcr}1-3 & 96-98 & 41.5 \\ 1-4 & 128-130 & 43.3 \\ 1-5 & 130-132 & 44.8 \\ 1-6 & 142-144 & 46.4 \\ 2-1 & 116-118 & 143.2 \\ 2-2 & 111-113 & 144.6 \\ 2-5 & 140-142 & 149.4\end{array}$

Hole 195

$\begin{array}{lcr}1-1 & 74-77 & 63.7 \\ 1-2 & 81-84 & 65.3 \\ 1-3 & 29-31 & 66.3 \\ 1-4 & 63-66 & 68.1 \\ 1-5 & 32-35 & 69.3 \\ 1-6 & 95-98 & 71.4 \\ 2-2 & 85-87 & 122.3 \\ 2-3 & 135-137 & 124.3 \\ 2-4 & 33-35 & 124.8 \\ 2-4 & 125-127 & 125.8\end{array}$

Hole 196

$\begin{array}{lcr}1-1 & 142-144 & 38.9 \\ 2-1 & 8-10 & 104.1 \\ 2-2 & 9-11 & 105.1 \\ 2-3 & 82-85 & 106.5 \\ 2-4 & 90-93 & 107.6 \\ 2-5 & 40-43 & 108.3\end{array}$

Hole 198A

$\begin{array}{ccc}1-2 & 142-144 & 93.4 \\ 1-4 & 100-102 & 96.0 \\ 1-5 & 80-82 & 97.3\end{array}$

TABLE 2 - Continued

\begin{tabular}{|c|c|c|}
\hline Core, Section & $\begin{array}{l}\text { Depth in Section } \\
(\mathrm{cm})\end{array}$ & $\begin{array}{l}\text { Depth Below Sea Floor } \\
\text { (m) }\end{array}$ \\
\hline $3-1$ & $51-53$ & 110.0 \\
\hline $3-6$ & $38-40$ & 117.4 \\
\hline $4-3$ & $108-110$ & 122.3 \\
\hline $4-4$ & $39-41$ & 122.9 \\
\hline $4-5$ & $103-105$ & 124.6 \\
\hline Hole 199 & & \\
\hline $1-1$ & $133-135$ & 58.8 \\
\hline $1-2$ & $63-65$ & 59.6 \\
\hline $1-3$ & $1-3$ & 60.5 \\
\hline $1-3$ & $14-16$ & 60.6 \\
\hline $1-4$ & $80-82$ & 62.8 \\
\hline $1-4$ & $110-112$ & 63.1 \\
\hline $1-5$ & $59-61$ & 64.1 \\
\hline $2-1$ & $100-102$ & 68.0 \\
\hline $2-3$ & $58-60$ & 70.6 \\
\hline $3-1$ & $135-137$ & 77.8 \\
\hline $3-3$ & $40-42$ & 79.9 \\
\hline $3-4$ & $75-77$ & 81.8 \\
\hline $3-5$ & $29-31$ & 82.8 \\
\hline $4-2$ & $100-102$ & 88.5 \\
\hline $4-2$ & $107-109$ & 88.6 \\
\hline $4-4$ & $70-72$ & 91.2 \\
\hline $4-5$ & $50-52$ & 92.5 \\
\hline $4-5$ & $100-102$ & 93.0 \\
\hline $5-1$ & $105-107$ & 144.1 \\
\hline $5-2$ & $103-105$ & 145.5 \\
\hline $5-4$ & $80-82$ & 148.3 \\
\hline $5-5$ & $80-82$ & 149.8 \\
\hline $5-6$ & $92-94$ & 151.4 \\
\hline $6-1$ & $47-49$ & 200.5 \\
\hline $6-1$ & $100-102$ & 201.0 \\
\hline $6-2$ & $90-92$ & 202.4 \\
\hline $6-2$ & $110-112$ & 202.6 \\
\hline $6-3$ & $58-60$ & 203.6 \\
\hline $6-4$ & $70-72$ & 205.2 \\
\hline $6-5$ & $80-82$ & 206.8 \\
\hline
\end{tabular}


TABLE 3

Results of X-Ray Diffraction Analyses from Hole 194

\begin{tabular}{|c|c|c|c|c|c|c|c|c|c|c|c|}
\hline \multicolumn{12}{|c|}{ Bulk Samples } \\
\hline Core & $\begin{array}{l}\text { Cored Interval } \\
\text { Below Sea } \\
\text { Floor (m) }\end{array}$ & $\begin{array}{l}\text { Sample Depth } \\
\text { Below Sea } \\
\text { Floor (m) }\end{array}$ & 串 & 这 & పేّ & $\frac{a 0}{\approx}$ & 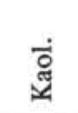 & $\stackrel{\text { J }}{\Sigma}$ & 方 & $\stackrel{\vec{E}}{\dot{\Xi}}$ & 总 \\
\hline \multirow[t]{4}{*}{1} & $37.5-47.0$ & 41.5 & 84.5 & 75.8 & 26.8 & 20.7 & - & 41.6 & 5.3 & 5.5 & - \\
\hline & & 43.3 & 88.1 & 81.4 & 27.1 & 23.8 & - & 39.3 & 4.0 & 4.3 & 1.4 \\
\hline & & 44.8 & 95.6 & 93.1 & 12.1 & 30.4 & - & 13.3 & - & 38.0 & 6.2 \\
\hline & & 46.4 & 90.1 & 84.6 & 28.7 & 24.0 & - & 33.7 & 5.0 & 8.6 & - \\
\hline \multirow[t]{3}{*}{2} & $142.0-151.5$ & 143.2 & 87.7 & 80.8 & 24.9 & 17.0 & 1.4 & 47.3 & 3.4 & 6.0 & - \\
\hline & & 144.6 & 87.7 & 80.8 & 27.5 & 12.2 & 2.0 & 52.9 & 2.7 & 2.7 & - \\
\hline & & 149.4 & 87.4 & 80.4 & 26.6 & 10.7 & 1.2 & 53.1 & 3.5 & 4.8 & - \\
\hline \multicolumn{12}{|c|}{$2-20 \mu \mathrm{m}$ Fractions } \\
\hline Core & $\begin{array}{c}\text { Cored Interval } \\
\text { Below Sea } \\
\text { Floor (m) }\end{array}$ & $\begin{array}{l}\text { Sample Depth } \\
\text { Below Sea } \\
\text { Floor }(\mathrm{m})\end{array}$ & 莕 & 官 & ةٌ & $\frac{m}{2}$ & $\begin{array}{l}\text { वं } \\
\text { ฮ্ }\end{array}$ & $\stackrel{\text { ֻூ }}{\Sigma}$ & 형 & 茫 & 完 \\
\hline \multirow[t]{4}{*}{1} & $37.5-47.0$ & 41.5 & 71.9 & 56.2 & 38.9 & 27.2 & - & 28.7 & 5.2 & - & - \\
\hline & & 43.3 & 77.9 & 65.4 & 36.7 & 30.4 & - & 28.8 & 4.1 & - & - \\
\hline & & 44.8 & 94.8 & 91.9 & 31.9 & 37.1 & - & 16.4 & 2.2 & 12.4 & - \\
\hline & & 46.4 & 80.9 & 70.2 & 39.3 & 28.3 & - & 27.5 & 4.9 & - & - \\
\hline \multirow[t]{3}{*}{2} & $142.0-151.5$ & 143.2 & 77.4 & 64.7 & 40.0 & 29.5 & - & 27.2 & 3.2 & - & - \\
\hline & & 144.6 & 75.3 & 61.3 & 43.8 & 22.2 & 0.6 & 29.1 & 3.0 & - & 1.3 \\
\hline & & 149.4 & 74.5 & 60.1 & 41.0 & 21.6 & - & 34.0 & 3.4 & - & - \\
\hline \multicolumn{12}{|c|}{$<2 \mu \mathrm{m}$ Fractions } \\
\hline Core & $\begin{array}{l}\text { Cored Interval } \\
\text { Below Sea } \\
\text { Floor }(\mathrm{m})\end{array}$ & $\begin{array}{l}\text { Sample Depth } \\
\text { Below Sea } \\
\text { Floor (m) }\end{array}$ & 苕 & 官 & 岂 & 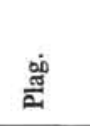 & 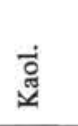 & $\stackrel{\mathscr{J}}{\Sigma}$ & $\frac{\text { ô }}{\text { E }}$ & $\stackrel{\text { D }}{\Sigma}$ & 竞 \\
\hline \multirow[t]{4}{*}{1} & $37.5-47.0$ & 41.5 & 80.2 & 69.0 & 16.3 & 5.7 & 3.0 & 43.4 & 6.2 & 25.5 & \\
\hline & & 43.3 & 82.4 & 72.5 & 21.0 & 9.4 & - & 39.0 & 7.2 & 23.4 & \\
\hline & & 44.8 & 78.5 & 66.4 & 4.7 & 5.9 & 1.1 & 4.4 & 1.2 & 82.6 & \\
\hline & & 46.4 & 82.4 & 72.4 & 20.8 & 10.6 & - & 33.4 & 6.3 & 29.0 & \\
\hline \multirow[t]{3}{*}{2} & $142.0-151.5$ & 143.2 & 84.6 & 75.9 & 21.9 & 9.8 & 4.8 & 40.8 & 4.2 & 18.6 & \\
\hline & & 144.6 & 86.2 & 78.4 & 25.9 & 8.9 & 3.6 & 43.1 & 3.3 & 15.3 & \\
\hline & & 149.4 & 85.3 & 77.0 & 26.4 & 8.9 & 2.4 & 42.3 & 4.0 & 15.9 & \\
\hline
\end{tabular}


TABLE 4

Results of X-Ray Diffraction Analyses from Hole 195

\begin{tabular}{|c|c|c|c|c|c|c|c|c|c|c|c|}
\hline \multicolumn{12}{|c|}{ Bulk Samples } \\
\hline Core & $\begin{array}{l}\text { Cored Interval } \\
\text { Below Sea } \\
\text { Floor }(\mathrm{m})\end{array}$ & $\begin{array}{l}\text { Sample Depth } \\
\text { Below Sea } \\
\text { Floor (m) }\end{array}$ & 产 & $\begin{array}{l}\dot{2} \\
\text { 응 } \\
\text { E }\end{array}$ & $\dot{\tilde{\Xi}}$ & $\frac{a}{\tilde{a}}$ & $\begin{array}{l}\dot{\tilde{g}} \\
\text { }\end{array}$ & $\stackrel{\oiiint}{\Sigma}$ & $\frac{\text { o }}{\text { ह }}$ & 苑 & 宫 \\
\hline 1 & $63.0-73.0$ & $\begin{array}{l}63.7 \\
65.3 \\
66.2 \\
68.1 \\
69.3 \\
71.4\end{array}$ & $\begin{array}{l}88.6 \\
86.5 \\
86.3 \\
86.9 \\
89.1 \\
87.1\end{array}$ & $\begin{array}{l}82.1 \\
78.9 \\
78.6 \\
79.5 \\
82.9 \\
79.8\end{array}$ & $\begin{array}{l}23.7 \\
28.8 \\
28.6 \\
29.5 \\
26.9 \\
23.9\end{array}$ & $\begin{array}{l}26.8 \\
22.5 \\
23.7 \\
27.9 \\
21.0 \\
18.8\end{array}$ & $\begin{array}{l}- \\
- \\
- \\
- \\
-\end{array}$ & $\begin{array}{l}37.6 \\
44.0 \\
40.7 \\
35.2 \\
42.6 \\
45.4\end{array}$ & $\begin{array}{l}4.1 \\
4.7 \\
4.6 \\
4.0 \\
4.1 \\
4.0\end{array}$ & $\begin{array}{l}7.8 \\
- \\
2.3 \\
3.4 \\
5.3 \\
7.9\end{array}$ & \\
\hline 2 & $120.0-129.5$ & $\begin{array}{l}122.3 \\
124.3 \\
124.8 \\
125.8 \\
\end{array}$ & $\begin{array}{l}87.8 \\
88.2 \\
87.7 \\
95.3 \\
\end{array}$ & $\begin{array}{l}80.9 \\
81.5 \\
80.8 \\
92.7\end{array}$ & $\begin{array}{l}27.8 \\
22.0 \\
25.2 \\
14.5 \\
\end{array}$ & $\begin{array}{l}17.5 \\
16.0 \\
16.6 \\
27.0\end{array}$ & $\begin{array}{l}- \\
1.8 \\
2.0 \\
3.4\end{array}$ & $\begin{array}{l}51.1 \\
40.2 \\
40.8 \\
30.8 \\
\end{array}$ & $\begin{array}{l}3.6 \\
1.7 \\
2.1 \\
- \\
\end{array}$ & $\begin{array}{l}\overline{18.3} \\
13.3 \\
24.3 \\
\end{array}$ & \\
\hline \multicolumn{12}{|c|}{$2-20 \mu \mathrm{m}$ Fractions } \\
\hline Core & $\begin{array}{c}\text { Cored Interval } \\
\text { Below Sea } \\
\text { Floor (m) }\end{array}$ & $\begin{array}{l}\text { Sample Depth } \\
\text { Below Sea } \\
\text { Floor }(\mathrm{m})\end{array}$ & 㟧 & 咅 & 㟧 & $\frac{\Xi}{2}$ & 㝏 & $\stackrel{\mathscr{0}}{\Sigma}$ & $\frac{\text { o̊ }}{\frac{1}{c}}$ & $\stackrel{\text { हू் }}{\Sigma}$ & 兘 \\
\hline 1 & $63.0-73.0$ & $\begin{array}{l}63.7 \\
66.3 \\
68.1 \\
69.3 \\
71.4\end{array}$ & $\begin{array}{l}79.8 \\
75.5 \\
79.5 \\
79.0 \\
74.9\end{array}$ & $\begin{array}{l}68.5 \\
61.7 \\
68.0 \\
67.3 \\
60.7\end{array}$ & $\begin{array}{l}35.8 \\
39.3 \\
37.2 \\
36.7 \\
39.5\end{array}$ & $\begin{array}{l}36.8 \\
27.1 \\
30.3 \\
29.3 \\
25.6\end{array}$ & & $\begin{array}{l}23.0 \\
28.1 \\
28.4 \\
29.4 \\
30.3\end{array}$ & $\begin{array}{l}4.4 \\
4.4 \\
4.1 \\
4.6 \\
4.6\end{array}$ & & $\begin{array}{l}\overline{1.1} \\
- \\
- \\
-\end{array}$ \\
\hline 2 & $120.0-129.5$ & $\begin{array}{l}122.3 \\
124.3 \\
124.8 \\
125.8 \\
\end{array}$ & $\begin{array}{l}77.6 \\
80.6 \\
77.9 \\
95.8\end{array}$ & $\begin{array}{l}64.9 \\
69.6 \\
65.5 \\
93.4\end{array}$ & $\begin{array}{l}36.3 \\
37.7 \\
39.5 \\
29.5 \\
\end{array}$ & $\begin{array}{l}25.4 \\
31.8 \\
28.5 \\
52.9\end{array}$ & & $\begin{array}{l}34.9 \\
28.2 \\
29.3 \\
17.6 \\
\end{array}$ & $\begin{array}{l}3.5 \\
2.3 \\
2.6 \\
- \\
\end{array}$ & & $\begin{array}{l}- \\
- \\
- \\
\end{array}$ \\
\hline \multicolumn{12}{|c|}{$<2 \mu \mathrm{m}$ Fractions } \\
\hline Core & $\begin{array}{l}\text { Cored Interval } \\
\text { Below Sea } \\
\text { Floor (m) }\end{array}$ & $\begin{array}{l}\text { Sample Depth } \\
\text { Below Sea } \\
\text { Floor (m) }\end{array}$ & 岕 & 家 & हี่ & 这 & 迅 & $\stackrel{\Xi}{\Sigma}$ & है & $\stackrel{\dot{0}}{\Sigma}$ & 家 \\
\hline 1 & $63.0-73.0$ & $\begin{array}{l}63.7 \\
66.3 \\
68.1 \\
69.3 \\
71.4\end{array}$ & $\begin{array}{l}83.2 \\
80.6 \\
81.3 \\
82.4 \\
84.5\end{array}$ & $\begin{array}{l}73.8 \\
69.6 \\
70.8 \\
72.4 \\
75.7\end{array}$ & $\begin{array}{l}19.9 \\
19.2 \\
20.6 \\
22.4 \\
25.3\end{array}$ & $\begin{array}{r}10.8 \\
9.6 \\
11.5 \\
11.6 \\
10.6\end{array}$ & $\begin{array}{l}- \\
4.6 \\
3.9 \\
2.3 \\
3.4\end{array}$ & $\begin{array}{l}37.5 \\
38.9 \\
40.6 \\
42.8 \\
43.0\end{array}$ & $\begin{array}{l}6.2 \\
5.9 \\
5.5 \\
5.6 \\
4.7\end{array}$ & $\begin{array}{l}25.6 \\
21.7 \\
17.9 \\
15.3 \\
13.0\end{array}$ & \\
\hline 2 & $120.0-129.5$ & $\begin{array}{l}122.3 \\
124.3 \\
124.8 \\
125.8\end{array}$ & $\begin{array}{l}82.6 \\
81.8 \\
84.7 \\
81.6\end{array}$ & $\begin{array}{l}72.8 \\
71.6 \\
76.1 \\
71.3\end{array}$ & $\begin{array}{r}21.8 \\
16.1 \\
26.2 \\
9.0\end{array}$ & $\begin{array}{r}8.7 \\
6.9 \\
12.9 \\
5.7\end{array}$ & $\begin{array}{l}2.9 \\
2.6 \\
5.4 \\
1.7\end{array}$ & $\begin{array}{l}27.0 \\
28.7 \\
43.7 \\
20.9\end{array}$ & $\begin{array}{l}2.1 \\
2.3 \\
3.7 \\
1.5\end{array}$ & $\begin{array}{r}37.6 \\
43.4 \\
8.1 \\
61.1\end{array}$ & \\
\hline
\end{tabular}


TABLE 5

Results of X-Ray Diffraction Analyses from Hole 196

\begin{tabular}{|c|c|c|c|c|c|c|c|c|c|c|c|c|c|c|c|}
\hline \multicolumn{16}{|c|}{ Bulk Samples } \\
\hline Core & $\begin{array}{l}\text { Cored Interval } \\
\text { Below Sea } \\
\text { Floor (m) }\end{array}$ & $\begin{array}{l}\text { Sample Depth } \\
\text { Below Sea } \\
\text { Floor (m) }\end{array}$ & 芯 & 官 & ల் & 芯 & 这 & $\frac{\dot{a j}}{\stackrel{a}{a}}$ & $\begin{array}{l}\dot{0} \\
\ddot{g}\end{array}$ & $\frac{\text { ֻூ }}{\Sigma}$ & อี & 䒕 & غू & 苞 & 竎 \\
\hline 1 & $37.5-47.0$ & 38.9 & 84.5 & 75.7 & - & 24.6 & - & 12.4 & 1.4 & 52.1 & 4.6 & 4.9 & - & - & - \\
\hline 2 & $104.0-110.0$ & $\begin{array}{l}104.1 \\
105.1 \\
106.5 \\
107.6 \\
108.3\end{array}$ & $\begin{array}{l}89.4 \\
89.4 \\
88.6 \\
89.5 \\
87.7\end{array}$ & $\begin{array}{l}83.5 \\
83.5 \\
82.2 \\
83.7 \\
80.8\end{array}$ & $\begin{array}{c}11.4 \\
- \\
- \\
- \\
-\end{array}$ & $\begin{array}{l}15.9 \\
11.2 \\
14.3 \\
20.8 \\
17.9\end{array}$ & $\begin{array}{c}8.9 \\
- \\
- \\
9.9 \\
13.9\end{array}$ & $\begin{array}{l}- \\
- \\
- \\
\overline{9} .6\end{array}$ & $\begin{array}{l}- \\
- \\
- \\
-\end{array}$ & $\begin{array}{l}13.6 \\
11.6 \\
15.7 \\
18.1 \\
30.5\end{array}$ & $\begin{array}{l}- \\
- \\
\overline{1.6} \\
2.7\end{array}$ & $\begin{array}{r}3.7 \\
\overline{3} .9 \\
10.0 \\
3.1\end{array}$ & $\begin{array}{l}44.6 \\
65.5 \\
52.6 \\
36.1 \\
11.8\end{array}$ & $\begin{array}{r}1.9 \\
11.7 \\
13.5 \\
- \\
-\end{array}$ & $\begin{array}{r}- \\
- \\
\overline{3} .6 \\
10.6\end{array}$ \\
\hline \multicolumn{16}{|c|}{ 2-20 $\mu \mathrm{m}$ Fractions } \\
\hline Core & $\begin{array}{l}\text { Cored Interval } \\
\text { Below Sea } \\
\text { Floor }(\mathrm{m})\end{array}$ & $\begin{array}{l}\text { Sample Depth } \\
\text { Below Sea } \\
\text { Floor (m) }\end{array}$ & 范 & 㻤 & गु & 䒕 & 造 & $\frac{\text { aी }}{a}$ & 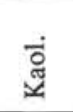 & 롤 & ฮี & 苑 & त्ञ & ฮี่ & 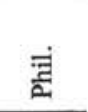 \\
\hline 1 & $37.5-47.0$ & 38.9 & 62.8 & 41.9 & & 36.2 & - & 20.5 & & 37.4 & 5.9 & - & & - & - \\
\hline 2 & $104.0-110.0$ & $\begin{array}{l}104.1 \\
105.1 \\
106.5 \\
107.6 \\
108.3\end{array}$ & $\begin{array}{l}73.6 \\
66.6 \\
67.1 \\
75.0 \\
70.1\end{array}$ & $\begin{array}{l}58.7 \\
47.9 \\
48.6 \\
60.9 \\
53.2\end{array}$ & & $\begin{array}{l}30.4 \\
24.8 \\
26.3 \\
31.8 \\
26.9\end{array}$ & $\begin{array}{l}38.0 \\
13.3 \\
14.5 \\
38.0 \\
11.4\end{array}$ & $\begin{array}{c}4.8 \\
- \\
- \\
- \\
10.4\end{array}$ & & $\begin{array}{l}20.5 \\
16.2 \\
21.3 \\
21.8 \\
24.9\end{array}$ & $\begin{array}{l}- \\
- \\
\overline{1.7}\end{array}$ & $\begin{array}{l}- \\
- \\
\overline{3.0} \\
-\end{array}$ & & $\begin{array}{r}6.4 \\
45.7 \\
38.0 \\
- \\
-\end{array}$ & $\begin{array}{c}- \\
- \\
\overline{5.5} \\
24.7\end{array}$ \\
\hline \multicolumn{16}{|c|}{$<2 \mu \mathrm{m}$ Fractions } \\
\hline Core & $\begin{array}{l}\text { Cored Interval } \\
\text { Below Sea } \\
\text { Floor }(\mathrm{m})\end{array}$ & $\begin{array}{l}\text { Sample Depth } \\
\text { Below Sea } \\
\text { Floor }(\mathrm{m})\end{array}$ & 范 & 官 & गु & 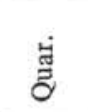 & 这 & $\frac{a 0}{a}$ & 晜 & 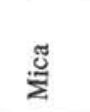 & 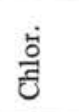 & 范 & त्ञ & $\dot{\Xi}$ & 㤩 \\
\hline 1 & $37.5-47.0$ & 38.9 & 83.2 & 73.7 & & 20.8 & - & 9.2 & 1.9 & 42.1 & 5.4 & 20.6 & - & - & - \\
\hline 2 & $104.0-110.0$ & $\begin{array}{l}104.1 \\
105.1 \\
106.5 \\
107.6 \\
108.3\end{array}$ & $\begin{array}{l}85.2 \\
85.9 \\
85.7 \\
87.2 \\
89.4\end{array}$ & $\begin{array}{l}76.9 \\
78.0 \\
77.7 \\
80.0 \\
83.5\end{array}$ & & $\begin{array}{r}8.3 \\
7.3 \\
10.8 \\
16.6 \\
17.8\end{array}$ & $\begin{array}{r}3.3 \\
\overline{5} \\
5.0 \\
12.0 \\
8.2\end{array}$ & $\begin{array}{l}- \\
- \\
- \\
\overline{4} .7\end{array}$ & $\begin{array}{l}1.9 \\
- \\
- \\
- \\
-\end{array}$ & $\begin{array}{r}20.4 \\
9.1 \\
9.3 \\
10.6 \\
18.5\end{array}$ & $\begin{array}{l}- \\
- \\
\overline{1.0} \\
2.7\end{array}$ & $\begin{array}{l}22.5 \\
21.8 \\
22.7 \\
26.6 \\
23.2\end{array}$ & $\begin{array}{l}43.5 \\
61.8 \\
49.1 \\
29.9 \\
13.6\end{array}$ & $\begin{array}{l}- \\
\overline{3.1} \\
- \\
-\end{array}$ & $\begin{array}{c}- \\
- \\
\overline{3.4} \\
11.4\end{array}$ \\
\hline
\end{tabular}


TABLE 6

Results of X-Ray Diffraction Analyses from Hole 198A

\begin{tabular}{|c|c|c|c|c|c|c|c|c|c|c|c|c|c|c|c|c|c|c|}
\hline \multicolumn{19}{|c|}{ Bulk Samples } \\
\hline Core & $\begin{array}{l}\text { Cored Interval } \\
\text { Below Sea } \\
\text { Floor }(\mathrm{m})\end{array}$ & $\begin{array}{l}\text { Sample Depth } \\
\text { Below Sea } \\
\text { Floor (m) }\end{array}$ & 岕 & 客 & లु & 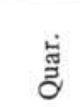 & $\dot{\ddot{b}}$ & 它 & $\frac{\infty}{2}$ & نं & ֻூ & $\frac{\text { है }}{E}$ & 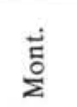 & बं & 苞 & छ் & 完 & $\frac{\pi}{5}$ \\
\hline 1 & $90.5-100.0$ & $\begin{array}{l}93.4 \\
96.0 \\
97.3\end{array}$ & $\begin{array}{l}84.8 \\
83.2 \\
83.3\end{array}$ & $\begin{array}{l}76.3 \\
73.7 \\
73.9\end{array}$ & $\overline{5}$ & $\begin{array}{l}26.7 \\
25.3 \\
27.1\end{array}$ & $\begin{array}{l}- \\
-\end{array}$ & $\begin{array}{r}13.6 \\
8.5 \\
9.1\end{array}$ & $\begin{array}{l}5.6 \\
5.0 \\
3.2\end{array}$ & $\begin{array}{l}\overline{-} \\
\overline{0.7}\end{array}$ & $\begin{array}{l}29.1 \\
26.7 \\
17.8\end{array}$ & $\begin{array}{l}3.5 \\
4.1 \\
3.2\end{array}$ & $\begin{array}{l}1.6 \\
2.9\end{array}$ & $\begin{array}{l}19.9 \\
24.6 \\
36.0\end{array}$ & $\begin{array}{l}- \\
-\end{array}$ & $\begin{array}{l}- \\
-\end{array}$ & $\begin{array}{l}- \\
-\end{array}$ & $\begin{array}{l}- \\
-\end{array}$ \\
\hline 2 & $109.5-119.0$ & $\begin{array}{l}110.0 \\
117.4\end{array}$ & $\begin{array}{l}85.5 \\
86.9\end{array}$ & $\begin{array}{l}77.4 \\
79.5\end{array}$ & - & $\begin{array}{l}25.5 \\
17.9\end{array}$ & - & $\begin{array}{l}21.1 \\
18.0\end{array}$ & $\begin{array}{l}6.9 \\
5.3\end{array}$ & $\begin{array}{l}2.3 \\
1.4\end{array}$ & $\begin{array}{l}18.2 \\
17.7\end{array}$ & $\begin{array}{l}1.4 \\
1.1\end{array}$ & $\begin{array}{l}5.0 \\
5.3\end{array}$ & $\begin{array}{l}19.7 \\
33.3\end{array}$ & - & - & - & - \\
\hline 4 & $119.0-125.0$ & $\begin{array}{l}122.3 \\
122.9 \\
124.6\end{array}$ & $\begin{array}{l}85.2 \\
88.8 \\
77.7\end{array}$ & $\begin{array}{l}76.9 \\
82.4 \\
65.1\end{array}$ & $\begin{array}{l}- \\
-\end{array}$ & $\begin{array}{l}18.7 \\
13.0 \\
51.0\end{array}$ & $\begin{array}{l}- \\
10.3 \\
16.0\end{array}$ & $\begin{array}{l}9.5 \\
1.7 \\
-\end{array}$ & $\overline{-}$ & $\begin{array}{l}- \\
-\end{array}$ & $\begin{array}{r}12.0 \\
12.1 \\
4.5\end{array}$ & $\begin{array}{l}- \\
-\end{array}$ & $\begin{array}{l}6.8 \\
5.5 \\
-\end{array}$ & $\begin{array}{r}37.3 \\
50.8 \\
7.5\end{array}$ & $\begin{array}{l}- \\
\overline{-} 1.1\end{array}$ & $\begin{array}{r}15.7 \\
6.6 \\
-\end{array}$ & - & - \\
\hline \multicolumn{19}{|c|}{$2-20 \mu \mathrm{m}$ Fractions } \\
\hline Core & $\begin{array}{l}\text { Cored Interval } \\
\text { Below Sea } \\
\text { Floor (m) }\end{array}$ & $\begin{array}{l}\text { Sample Depth } \\
\text { Below Sea } \\
\text { Floor }(\mathrm{m})\end{array}$ & 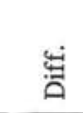 & 官 & లु & 岕 & : & 官 & $\stackrel{0}{\approx}$ & 通 & $\frac{\mathscr{J}}{\Sigma}$ & $\frac{\dot{0}}{\text { ह }}$ & $\stackrel{\dot{0}}{\Sigma}$ & हैं & 总 & ఏ & 咅 & $\frac{\pi}{5}$ \\
\hline 1 & $90.5-100.0$ & $\begin{array}{l}93.4 \\
96.0 \\
97.3\end{array}$ & $\begin{array}{l}69.1 \\
63.5 \\
65.6\end{array}$ & $\begin{array}{l}51.6 \\
43.0 \\
46.3\end{array}$ & $\begin{array}{l}- \\
-\end{array}$ & $\begin{array}{l}41.7 \\
42.1 \\
42.3\end{array}$ & $\begin{array}{l}- \\
- \\
-\end{array}$ & $\begin{array}{l}18.9 \\
15.1 \\
13.4\end{array}$ & $\begin{array}{l}7.1 \\
8.5 \\
8.5\end{array}$ & $\begin{array}{l}-\cdot \\
-\end{array}$ & $\begin{array}{l}28.2 \\
30.2 \\
31.4\end{array}$ & $\begin{array}{l}4.0 \\
4.2 \\
4.4\end{array}$ & $\begin{array}{l}- \\
-\end{array}$ & $\begin{array}{l}- \\
-\end{array}$ & $\begin{array}{l}- \\
- \\
-\end{array}$ & $\begin{array}{l}- \\
-\end{array}$ & $\begin{array}{l}- \\
-\end{array}$ & $\begin{array}{l}\bar{P} \\
\mathrm{~T}\end{array}$ \\
\hline 3 & $109.5-119.0$ & $\begin{array}{l}110.0 \\
117.4\end{array}$ & $\begin{array}{l}72.3 \\
74.0\end{array}$ & $\begin{array}{l}56.6 \\
59.4\end{array}$ & - & $\begin{array}{l}37.6 \\
27.9\end{array}$ & - & $\begin{array}{l}28.7 \\
33.0\end{array}$ & $\begin{array}{r}10.3 \\
9.3\end{array}$ & $\begin{array}{l}- \\
-\end{array}$ & $\begin{array}{l}21.1 \\
21.9\end{array}$ & $\begin{array}{l}2.2 \\
1.6\end{array}$ & - & - & - & $\overline{6.3}$ & - & - \\
\hline 4 & $119.0-125.0$ & $\begin{array}{l}122.3 \\
122.9 \\
124.6\end{array}$ & $\begin{array}{l}64.0 \\
64.4 \\
78.1\end{array}$ & $\begin{array}{l}43.8 \\
44.4 \\
65.7\end{array}$ & $\begin{array}{l}- \\
-\end{array}$ & $\begin{array}{l}30.6 \\
31.3 \\
43.6\end{array}$ & $\begin{array}{l}- \\
\overline{32.2}\end{array}$ & $\begin{array}{c}17.7 \\
11.8 \\
-\end{array}$ & $\begin{array}{l}- \\
-\end{array}$ & $\overline{-}$ & $\begin{array}{r}18.5 \\
20.9 \\
1.9\end{array}$ & $\begin{array}{l}- \\
- \\
-\end{array}$ & $\overline{-}$ & $\begin{array}{l}- \\
-\end{array}$ & $\begin{array}{c}- \\
\overline{22.2}\end{array}$ & $\begin{array}{c}33.3 \\
25.7 \\
-\end{array}$ & $\overline{0} \overline{0.3}$ & A \\
\hline \multicolumn{19}{|c|}{$<2 \mu \mathrm{m}$ Fractions } \\
\hline Core & $\begin{array}{l}\text { Cored Interval } \\
\text { Below Sea } \\
\text { Floor (m) }\end{array}$ & $\begin{array}{l}\text { Sample Depth } \\
\text { Below Sea } \\
\text { Floor (m) }\end{array}$ & 范 & 宫 & $\frac{\dot{0}}{\tilde{J}}$ & ప્ّ & 站 & $\frac{\dot{0}}{4}$ & 胫 & $\begin{array}{l}\dot{\bar{g}} \\
\check{\check{g}}\end{array}$ & $\stackrel{\check{\Sigma}}{\grave{\Sigma}}$ & 官 & है & ले & 官 & घ் & 竎 & $\frac{\pi}{5}$ \\
\hline 1 & $90.5-100.0$ & $\begin{array}{l}93.4 \\
96.0 \\
97.3\end{array}$ & $\begin{array}{l}88.2 \\
86.5 \\
85.4\end{array}$ & $\begin{array}{l}81.6 \\
78.9 \\
77.3\end{array}$ & $\begin{array}{l}- \\
\overline{-}\end{array}$ & $\begin{array}{l}14.9 \\
15.2 \\
13.4\end{array}$ & $\begin{array}{l}- \\
-\end{array}$ & $\begin{array}{l}9.4 \\
6.2 \\
3.6\end{array}$ & $\begin{array}{l}3.5 \\
2.8 \\
2.0\end{array}$ & $\begin{array}{l}\overline{3.1} \\
0.9\end{array}$ & $\begin{array}{l}14.6 \\
21.2 \\
22.3\end{array}$ & $\begin{array}{l}2.8 \\
2.1 \\
4.3\end{array}$ & $\begin{array}{l}29.8 \\
14.9 \\
11.7\end{array}$ & $\begin{array}{l}25.0 \\
34.5 \\
41.8\end{array}$ & $\begin{array}{l}- \\
-\end{array}$ & $\begin{array}{l}- \\
-\end{array}$ & $\overline{-}$ & - \\
\hline 3 & $109.4-119.0$ & $\begin{array}{l}110.0 \\
117.4\end{array}$ & $\begin{array}{l}85.8 \\
85.8\end{array}$ & $\begin{array}{l}77.9 \\
77.8\end{array}$ & - & $\begin{array}{l}16.2 \\
11.8\end{array}$ & - & $\begin{array}{l}9.8 \\
9.4\end{array}$ & $\begin{array}{l}2.9 \\
3.3\end{array}$ & $\begin{array}{l}1.5 \\
1.4\end{array}$ & $\begin{array}{r}17.0 \\
9.8\end{array}$ & $\begin{array}{l}2.1 \\
0.7\end{array}$ & $\begin{array}{l}30.5 \\
22.2\end{array}$ & $\begin{array}{l}20.0 \\
41.5\end{array}$ & - & - & - & - \\
\hline 4 & $119.0-125.0$ & $\begin{array}{l}122.3 \\
122.9 \\
124.6\end{array}$ & $\begin{array}{l}88.3 \\
88.1 \\
89.0\end{array}$ & $\begin{array}{l}81.7 \\
81.4 \\
82.8\end{array}$ & $\begin{array}{l}- \\
- \\
-\end{array}$ & $\begin{array}{l}16.5 \\
11.7 \\
10.2\end{array}$ & $\begin{array}{c}- \\
\overline{50.0}\end{array}$ & $\begin{array}{l}6.5 \\
- \\
-\end{array}$ & $\begin{array}{l}- \\
-\end{array}$ & - & $\begin{array}{r}11.0 \\
7.0 \\
4.0\end{array}$ & $\begin{array}{l}- \\
-\end{array}$ & $\begin{array}{r}20.0 \\
18.7 \\
5.1\end{array}$ & $\begin{array}{l}41.7 \\
60.4 \\
10.1\end{array}$ & $\begin{array}{c}- \\
\overline{-} \\
20.6\end{array}$ & $\begin{array}{l}4.4 \\
2.2 \\
-\end{array}$ & $\begin{array}{l}- \\
-\end{array}$ & - \\
\hline
\end{tabular}

aU-1 peaks at $4.21 \AA$ (narrow), $5.35 \AA$ (narrow), $4.61 \AA$ (narrow), and $3.80 \AA$ (broad). $\mathrm{P}=$ present, $\mathrm{T}=$ trace, $\mathrm{A}=$ abundant. 
TABLE 7

Results of X-Ray Diffraction Analyses from Hole 199

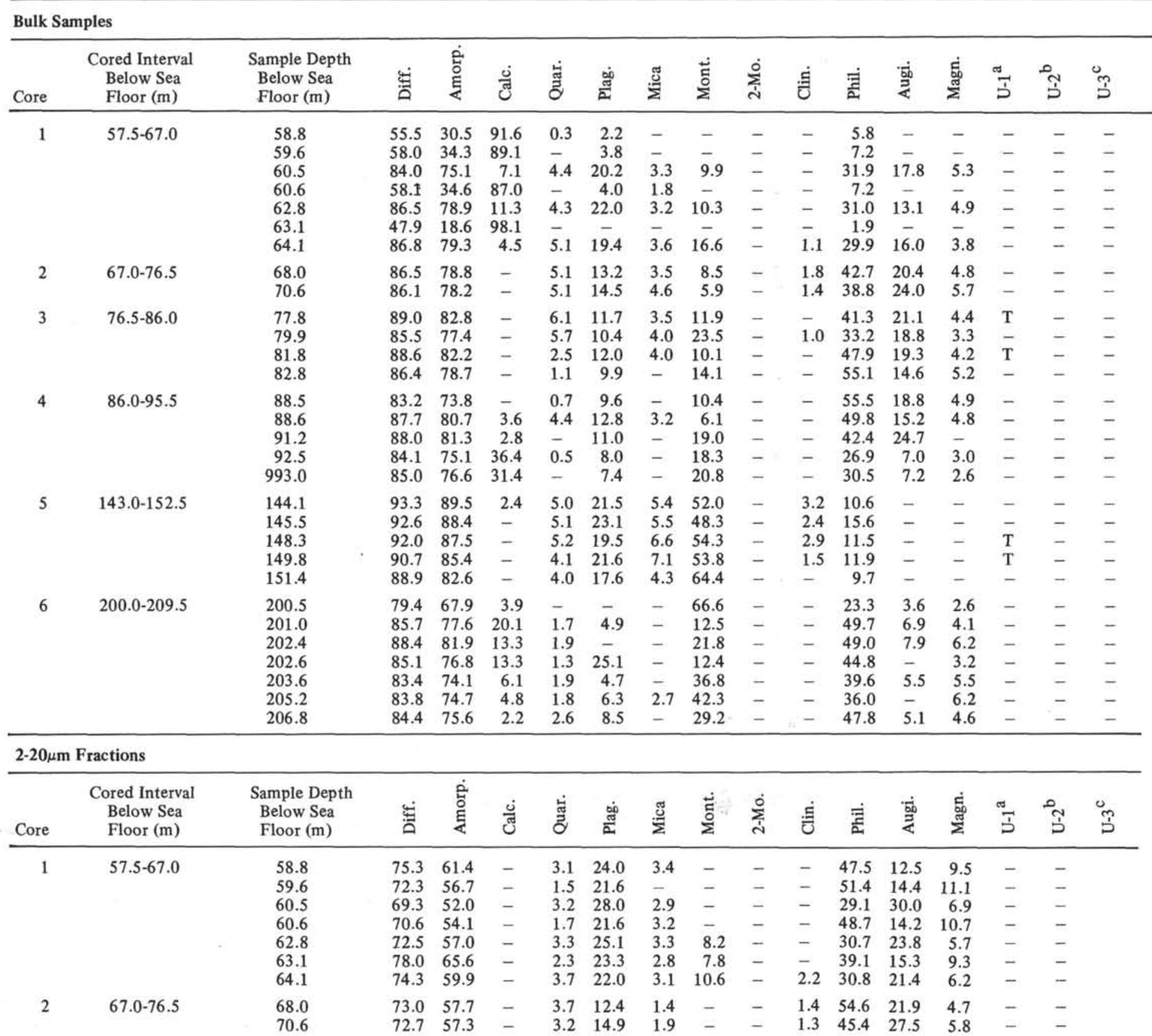


TABLE 7 - Continued

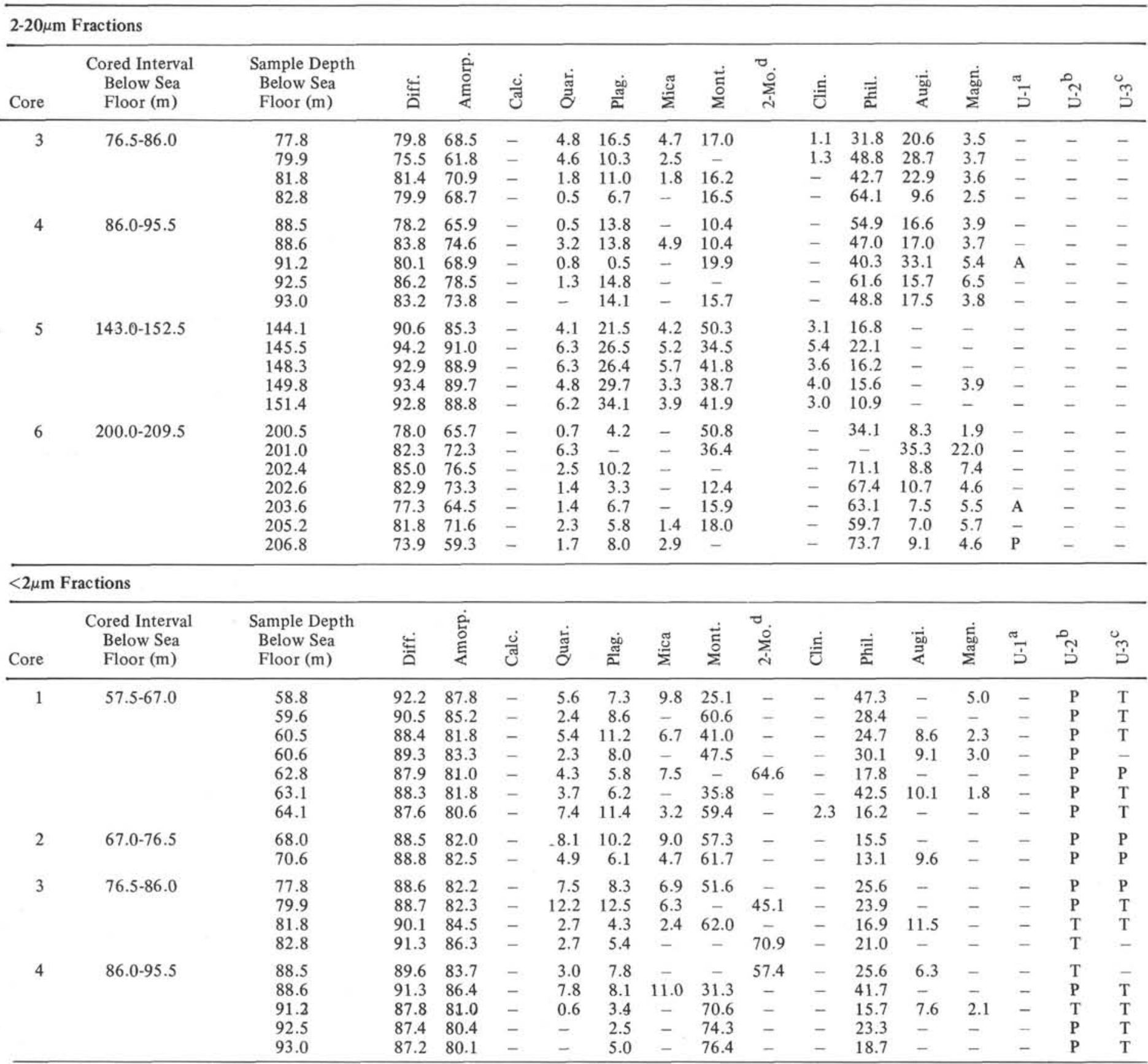


TABLE 7 - Continued

\begin{tabular}{|c|c|c|c|c|c|c|c|c|c|c|c|c|c|c|c|c|c|}
\hline \multicolumn{18}{|c|}{$<2 \mu \mathrm{m}$ Fractions } \\
\hline Core & $\begin{array}{l}\text { Cored Interval } \\
\text { Below Sea } \\
\text { Floor (m) }\end{array}$ & $\begin{array}{l}\text { Sample Depth } \\
\text { Below Sea } \\
\text { Floor }(\mathrm{m})\end{array}$ & 唆 & 容 & లु & है & 递 & 荧 & ह & $\sum_{i}^{0}$ & ఏ் & 竎 & 常 & 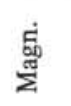 & $\stackrel{\pi}{3}$ & ث̃ & $\stackrel{\tilde{s}}{\dot{s}}$ \\
\hline \multirow[t]{5}{*}{5} & $143.0-152.5$ & 144.1 & 87.8 & 81.0 & - & 1.9 & 5.1 & 6.0 & 87.0 & - & - & - & - & - & - & $\mathrm{T}$ & $\mathrm{T}$ \\
\hline & & 145.5 & 87.6 & 80.6 & - & 2.1 & 5.2 & 7.1 & 85.6 & - & - & - & - & - & - & $\mathrm{T}$ & $T$ \\
\hline & & 148.3 & 85.7 & 77.6 & - & 1.7 & 3.4 & 7.1 & 87.8 & - & - & - & - & - & - & $\mathrm{T}$ & P \\
\hline & & 149.8 & 82.0 & 71.9 & - & 1.0 & 2.3 & 6.9 & 87.3 & - & - & 2.5 & - & - & - & $\mathrm{T}$ & $\mathrm{T}$ \\
\hline & & 151.4 & 83.1 & 73.6 & - & 0.8 & 2.7 & 5.8 & 90.8 & - & - & - & - & - & - & $\mathrm{T}$ & $\mathrm{T}$ \\
\hline \multirow[t]{7}{*}{6} & $200.0-209.5$ & 200.5 & 79.4 & 67.8 & - & - & - & - & 35.1 & 61.3 & - & 3.6 & - & - & - & P & $\mathrm{T}$ \\
\hline & & 201.0 & 91.4 & 86.6 & - & 2.7 & 5.5 & - & 64.2 & - & - & 27.6 & - & - & - & P & $T$ \\
\hline & & 202.4 & 90.4 & 84.9 & - & 2.3 & - & - & 71.2 & - & - & 26.5 & - & - & - & $P$ & $P$ \\
\hline & & 202.6 & 90.1 & 84.5 & - & 1.2 & 5.7 & - & 51.2 & 18.6 & - & 20.6 & - & 2.7 & - & $\mathrm{T}$ & $\mathrm{T}$ \\
\hline & & 203.6 & 86.6 & 79.0 & - & 1.0 & - & 4.9 & 80.7 & - & - & 13.3 & - & - & - & $\mathrm{P}$ & $\mathrm{T}$ \\
\hline & & 205.2 & 86.9 & 79.5 & - & 0.8 & 3.9 & - & 77.9 & - & - & 14.9 & - & 2.4 & - & P & $T$ \\
\hline & & 206.8 & 89.4 & 83.4 & - & 0.8 & 4.7 & 5.0 & 66.5 & - & - & 20.0 & - & 3.1 & - & P & $\mathrm{T}$ \\
\hline
\end{tabular}

aU-1 peak at $4.21 \AA$ (narrow). $\mathrm{A}=$ abundant, $\mathrm{T}=$ trace, $\mathrm{P}=$ present

bU-2 peaks at $3.003 \AA, 2.813 \AA$, and $6.01 \AA$.

cU-3 broad peak at $3.535 \AA$.
$\mathrm{d}_{2}$-Mo. $=\mathrm{A}$ second variety of montmorillonite with different expansion characteristics was detected. 